\title{
INTEGRATED PERFORMANCE MEASUREMENT SYSTEM
}

\author{
O. Ahmed* \\ Department of Economics Industrial Engineering and Management, Faculty of the Management, \\ Technical University, Sofia Bulgaria
}

\begin{abstract}
The aim of the work is to create an integrated and practical applicable system for assessing SME's performance. To achieve this goal, research, analytical and design tasks are solved. As a result, an innovative solution has been developed for integrated assessment and management of the SME's activities. The Characteristics of Performance Measurement and Factors Influencing Performance Measurement in SMEs are studied. Concept and objectives of the Integrated performance measurement system are developed. The structure and configuration of the performance measurement system are conceptualized. At the end of the work, conclusions are drawn about the application of the developed conceptual solution in practice.
\end{abstract}

Key words: Performance measurement; Performance Measurement in SMEs; Integrated performance measurement.

\section{INTRODUCTION}

Businesses are facing tough challenges to succeed in a global competitive market. Customer demand is changing rapidly in terms of sophistication of the products and services they require. As a result, companies need to become more responsive to customers and market needs, with a greater number of customer specific products and/or services, more flexible processes, suppliers and resources co-ordinated through a number of organisations along the supply chain, whilst reducing costs. In order to proactively respond to these challenges, management requires upto-date and accurate performance information on its business. This performance information needs to be integrated, dynamic, accessible and visible to aid fast decision-making to promote a pro-active management style leading to agility and responsiveness.

The main objective of this work is to introduce and develop the concept of Performance Management as a key business process and focus on the criticality of the Performance Measurement System embedded within. It presents a reference model for the Performance

\footnotetext{
*Correspondence to: Otman Otman Ahmed, Department of Economics Industrial Engineering and Management, Faculty of the management, Technical University in Sofia.,N8 Kliment Ohridski Blvd., 1756 Sofia, sdim@tu-sofia.bg
}

Measurement System which is based on industry best practices.

\section{Performance measurement systems in SMEs}

In recent years, literature has identified the increasing complexity of small and mediumsized enterprises (SMEs) and highlighted their sensitivity to differences in managerial culture and management systems. Research has shown that performance measurement systems (PMSs) could play an important role in supporting managerial development in these companies.

In fact, it has often been said [7, 27] that the critical factors for the success of these SMEs can mostly be found in the attributes of a model of a company whose success basically depends on the figure of the entrepreneurowner, who is personally responsible for managing the activities of the company. This model is characterized by: flexibility and an ability to react quickly and adapt to the competitive and changing environment; organizational processes which are not very structured or 'engineered'; significant concentration of decision-making processes in the entrepreneur-owner; focus on technical aspects and production; and the existence of specialist and tacit knowledge that is essentially technological and evolves through learning processes based on learning by doing $[18,21,22]$. 
Small and large firm are fundamentally different from each other in three central aspects: uncertainty, innovation and evolution; literature underlines that the central distinction between large and small firms is the greater external uncertainty of the environment in which the small firm operates, together with the greater internal consistency of its motivations and actions [29, 31].

The increasing importance of continuous improvement has led many researchers to point out that PMSs might actually be needed to support continuous improvement processes [2, $3,20,23,25,26]$.

In addition, SMEs tend to have poor strategic planning and do not fully understand what their critical success factors are [11]. The process of designing a PMS forces a company to do strategic planning, and implementing and using it highlights the gaps between the company's current performance and its objectives. Consequently, the PMS helps the company set future objectives and plan any necessary improvement processes [30]). As Kaplan and Norton [19] and Lynch and Cross [20] write, implementing a PMS favours the creation of learning processes and, therefore, agreement on which processes need to be improved.

\subsection{Characteristics of Performance Measurement in SMEs}

After a thorough literature review five common characteristics were identified.

- The difficulty in involving SMEs in performance measurement projects. Moreover, the companies that do take part in these projects rarely continue on to the last phase because of the lack of time available for nonoperational activities and the poor involvement of the entrepreneurs or top managers in the PM project [30].

- The studies indicate that SMEs either do not use any PM model or they use models incorrectly. Many companies often implement only some parts of a general model, while others modify the models without carefully considering the changes made. In other words, they eliminate some dimensions without first carefully understanding and analysing the characteristics of the model and the company.

- SMEs' approach to performance measurement is informal, not planned and not based on a predefined model; performance measurement is introduced to solve specific problems and the PMS grows out of this process spontaneously rather than as a result of planning [3].
- SMEs have limited resources for data analysis. Data are gathered and the processes analysed in an imprecise way, and this unformalized approach increases the ambiguity of the measurement objectives. The information is then presented in the same way: SMEs usually use tables rather than graphs, making it difficult to interpret the information $[1,3]$.

\subsection{Factors Influencing Performance Measurement in SMEs}

Many researchers state that the specific characteristics of SMEs can be obstacles to the implementation and use of a PMS. These characteristics are briefly described in the following paragraphs.

- Lack of human resources. SMEs have limited human resources. All the staff are involved in the activities of managing daily work, and have no extra time for additional activities, such as implementing a PMS [3, 14, 17, 24, 28, 30].

- Managerial capacity. Technical excellence in products and operational processes is often perceived as the only key critical factor in SMEs. Very often, employees occupy different positions at the same time, the organizations are flat, and though the entrepreneur is in charge of both operational and managerial functions, he/she usually neglects the managerial activities [21].

- Limited capital resources. The impact of the resources needed to implement a PMS is proportionally more onerous in SMEs than in large companies $[3,9,10,14,17,26,28]$. Moreover, the absence of affordable software platforms that focus on the specific needs of SMEs further obstructs the introduction of PMSs in these companies [5].

- Reactive approach. SMEs are characterized by poor strategic planning and their decisionmaking processes are not formalized. The lack of explicit strategies and methodologies to support the control process promotes both a short-term orientation and a reactive approach to managing the company's activities [8,21]

- Tacit knowledge and little attention given to the formalization of processes. One of the main barriers to organizational development in SMEs is the lack of a managerial system and formalized management of the processes. Furthermore, since knowledge is mainly tacit and context-specific, the information required to implement and use a PMS is difficult to gather $[18,21,22]$.

- Misconception of performance measurement. Hudson et al. [15] underlines that a PMS can only be effectively implemented and used when the company perceives the benefits of 
the PMS. SMEs often do not understand the potential advantages of implementing a PMS; these systems are perceived as a cause of bureaucratization and an obstacle to the flexibility of SMEs [16, 17, 24].

The limited resources of SMEs require approaches and models that respond to their specific needs and are efficient and easy to implement. The employees involved in implementing and using PMSs must clearly understand the short- and long-term advantages in order to maintain their enthusiasm and commitment [13]. PMSs for SMEs must be dynamic and flexible in order to respond to the needs of these companies, but at the same time they must also be structured to a certain degree in order to favour activity planning [3, 12, 15]. Though the design of PMSs for SMEs must consider strategy, there must also be a strong focus on operational aspects, since traditionally these are the aspects that are critical for the success of SMEs.

All these factors underline the differences between SMEs and big companies and the need for a different approach to PM in SMEs. Moreover, these factors could be useful in the study of the dimensions of PMSs for SMEs.

\section{Concept and objectives of the integrated performance measurement system}

Many authors have focused attention on how organizations can design more appropriate measurement systems. Hudson et al. [15] identify nine requirements for an effective PM development process. These include (1) need evaluation/existing PM audit, (2) key user involvement, (3) strategic objective identification, (4) performance measure development, (5) periodic maintenance structure, (6) top management support, (7) full employee support, (8) clear and explicit objectives, and (9) set timescales. Identifying the procedures for developing strategic PM systems is rather more problematic, as these will vary between processes. However, to ensure strategic alignment, a procedure for identifying strategic objectives should be included. A method for developing the measures is necessary, along with a procedure for maintaining the new PM system $[15,26]$

\subsection{The structure and configuration of the performance measurement system}

The literature review revealed two critical considerations with respect to the structure and configuration of Performance Measurement Systems. These are: Integrity of the System; Deployment

\subsection{Integrity of the System}

Integrity refers to the ability of the performance measurement system to promote integration between various areas of the business. Previous research on manufacturing systems integration identified the value of the Viable Systems Model [4, 6] as a framework for assessing a systems integrity. The theory behind the Viable Systems Model (VSM) is that for any system to be viable it must have five sub-systems. The VSM identifies these five sub-systems as System 1, System 2, System 3, System 4, System 5 and the Meta System which is a combination of the Systems 3, 4 and 5. These systems are discussed below. System 1 are the operational units which produces the goods or services. In other words it represents the productive function of the organisation. It could be interpreted that system 1 consists of Business Processes such as Order Fulfillment, Product Development, Get Order, and so on. From a performance measurement systems perspective system 1 will consists of performance measures which objectively measure the performance of an individual Business Process.

System 2 is the supervisory system which coordinates the activities of operational units. This system represents the Business Process which co-ordinates the activities of system one.

System 3 represents the tactical management system which manages the operations of the systems 1 and 2 by setting targets and priorities. Also, in terms of the meta system it would be the system for the implementation of change. This is the management system which is responsible for the performance of business processes and activities in line with the requirements of the higher level systems. From an performance measurement systems perspective this is the system which deploys the strategic policies and priorities. It is therefore responsible for deploying targets and priorities to the measures under systems 1 and 2

System 4 is the developmental system which concerns itself with the external environment and therefore the future. Its focus is on improvement. In terms of the meta system this is the system which identifies the changes necessary to the lower level systems, i.e. systems 1,2 and 3 . This effectively is the benchmarking system which by focusing externally identifies the improvement gaps. The performance measures used in this system tends to be externally focused and comparative, e.g. delivery performance with 
respect to the competitors or market requirements. In addition to measuring the performance gaps with respect to the external environment, one other key function of this system is the prioritisation of the these gaps in line with the corporate and strategic objectives set by the higher level system 5 .

The Meta System is the combination of systems 5, 4 and 3 which is responsible for identifying and managing change. System 5 sets policy and direction, system 4 identifies the necessary changes and system 3 implements these changes.

\subsection{Deployment}

Deployment refers to the deployment of business objectives and policies throughout the hierarchical structure of the organisation as illustrated in Figure 1. Objective of deployment, in this context, is to ensure that:

- Performance measures used at various levels of the organisation reflect the business objectives and policies.

- Deployment is consistent through the hierarchy of the organisation.

- Deployment is relevant and correct with respect to the impact and influence of individual business areas (i.e. processes, functions and activities).

\section{A reference model}

The reference model developed by the author is based upon the two facets of the performance measurement systems as identified above. These are: Integrity; Deployment

A reference model has been developed following a study of best practices. At each level of the structure four key factors are considered, these are: Stakeholders, Control criteria, External measures, Improvement objectives and Internal measures. The reference model places emphasis on the amplification, transduction and attenuation of performance objectives between these levels.

The reference model uses this basic structure to integrate a following concepts into a single framework:

- Policy Deployment. The deployment of corporate and stakeholders objectives throughout the organisation.

- Competitive Criteria and Benchmarking. The definition of key competitive factors and position of the Business and the Business Units within its competitive environment.

- Process Orientation. Focusing on key business processes to manage business performance.

- Normative Planning. The measurement methodology which differentiates between Actuality, Capability and Potentiality.

- Active Monitoring. The use of proactive performance measures rather then reactive measures.

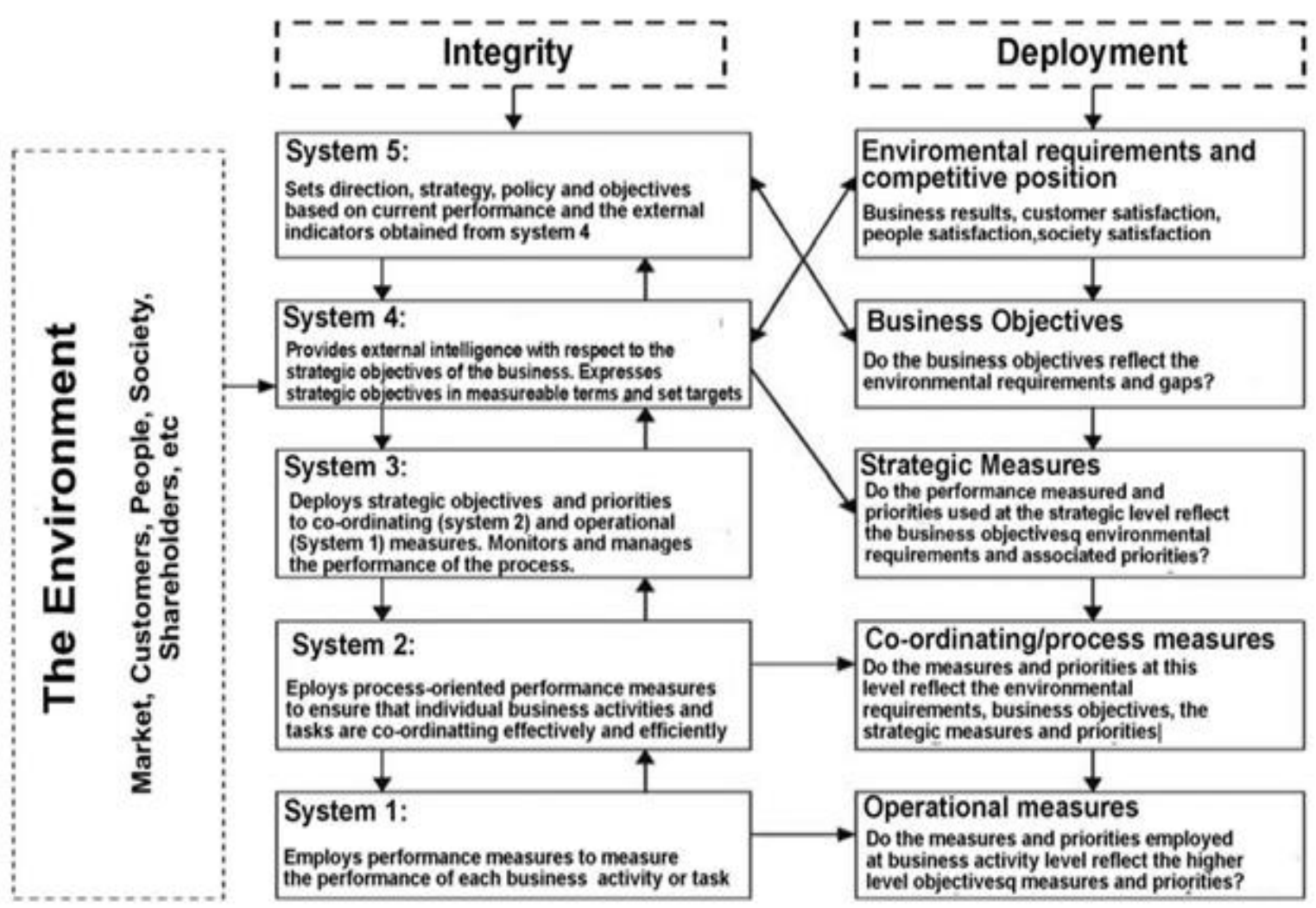

Figure 1. A Reference model 


\section{The audit method}

Having identified and defined the key characteristics of an integrated performance

measurement system (i.e. Integrity and Deployment), the author has developed a method for auditing the Integrity and Deployment of the performance measurement system as defined in the reference model.

The audit process consists of three phases. These are: Data Collection; Integrity Audit; Deployment Audit.

\subsection{Data Collection Phase}

The Data Collection Phase, using a workbook developed by the author, collects all information relevant to the Performance Management Process of the business. The information collected includes:

- Identification of Business Units (BU) within the business.

- The market requirements in terms of qualifiers and differentiators for each BU.

- The development plans or objectives for the business or each of its BU's.

- The performance measures used and reviewed by the executive management team within the business, i.e. the Strategic Performance Measures

- The performance measures used and reviewed within each function of the business, i.e. the Functional or Operational Performance Measures.

- Personal objectives and any associated incentive scheme for the executives, managers, supervisors and operational personnel

- Review, reporting and performance responsibilities associated with measures used at all levels.

Once the data is collected it is then analysed to conduct the Integrity and Deployment audits respectively.

\subsection{Integrity Audit}

In order to conduct the Integrity Audit in a comprehensive, rigorous and repeatable manner, the author has compiled a checklist to search for objective evidence supporting the existence of all five systems within the VSM. Following the analysis of this data against this checklist various gaps are identified with respect to the completeness and integrity of the performance measurement system.

\subsection{The Deployment Audit}

During the process of creating the model it became apparent that most businesses, small or large, consists of discrete business units which in turn may be treated as stand alone businesses.
In the first instance the deployment audit requires the identification of the individual SBU's and their strategic requirements in terms of the qualifiers and the differentiators. The performance measurement system should ensure that the priorities between the above two criteria are balanced and appropriately managed in a dynamic environment.

In order to audit the deployment of the performance measurement system a there stage audit method has been developed:

- Stage one focuses on to the requirements of each SBU environment and through a series of matrices assesses the deployment of environmental requirements through business objectives to strategic and functional performance measures.

- Stage two focuses on to the business objectives and through a series of similar set of matrices assesses their deployment through the strategic performance measures to functional levels.

- Stage three focuses on the deployment of strategic performance measures to the functional levels, again through the use of similar matrices.

The audit process is designed to be rigorous in order to ensure repeatability. A scoring system has been devised to measure the strength of deployment which results in a rigorous approach and repeatable conclusions.

\section{CONCLUSION}

The research and development effort of the author is aimed at development of practical tools and techniques to facilitate more scientific and precise management of business performance. More specifically the research and development work presented in this paper may be summarised as follows:

- The research work carried out by author as part of a overall work on dissertation focusing on performance measurement systems identified the need to study Performance Management as a process.

- The Performance Management Process is seen as a closed loop control system which deploys policy and strategy, and obtains feedback from various levels in order to manage the performance of the business.

- The Performance Measurement System is the information system which is at the heart of the Performance Management Process and it is of critical importance to the effective and efficient functioning of the Performance Management System.

- Research Identified two critical elements with respect to the content and structure of the 
Performance Measurement System, these are: Integrity and Deployment.

- The Reference Model developed for Integrated Performance Measurement Systems provides a framework against which performance measurement system can be designed and audited.

- The Audit Methods developed to assess the Integrity and Deployment of the performance measurement system provide simple but rigorous and repeatable tools which may be used to improve the effectiveness and efficiency of the Performance Management Process.

\section{REFERENCES}

1. Antonelli, V., Cerbioni, F., \& Parbonetti, A., The rise of cost accounting: evidence from Italy. Accounting, Business \& Financial History, 12(3): 461-486, 2002.

2. Atkinson, A. and Waterhouse, J.H., A stakeholder approach to strategic performance measurement. Sloan Management Review, 38: 25-37, 1997.

3. Barnes, M., Dickinson, T., Coulton, L., Dransfield, S., Field, J., Fisher, N, Saunders, I. and Shaw, D., A new approach to performance measurement for small to medium enterprises. In Proceedings of the Performance Measurement - Theory and Practice Conference, Cambridge, 14-17 July, 1998.

4. Beer, S.,. The viable system model: Its provenance, development, methodology and pathology. Journal of the operational research society, 35(1): 7-25, 1984.

5. Bititci, U.S., Turner, T, Nudurupati, S.S. and Creighton, S., Web enabled measurement systems - management implications, International Journal of Operations and Production Management, 22: 1273-1287, 2002.

6. Blenkinsop, S. A., Organisational aspects of information processing systems (Doctoral dissertation, SA Blenkinsop, 1993.

7. Bridge S., O'Neill, K. and Cromie, S., Understanding Enterprise, Entrepreneur ship \& Small Business. London: Macmillan, 1998.

8. Brouthers, K., Andriessen, F. and Nicolaes, I., Driving blind: strategic decision-making in small companies. Long Range Planning, 31: 130-138, 1998.

9. Burns, P. and Dewhurst, J., Small Business and Entrepreneurship. London: Macmillan, 1996.

10.Ghobadian, A. and Gallear, D., TQM and organisation size. International Journal of Operations and Production Management, 17: 121-163, 1997.
11.Greatbanks, R., \& Boaden, R., Can SMMEs afford to measure performance. In Conference Proceedings Performance Measurement-Theory and Practice,Vol. 1: 117-124, July, 1998.

12.Hudson, M. and Smith, D., Running before walking: the difficulties of developing strategic performance measurement systems in SMEs. In the Proceedings of the 7th International Annual EurOMA Conference, Ghent, 4-7 June, 2000.

13.Hudson, M., Bennett, J., Smart, PA., Bourne, M., Performance measurement for planning and control in SMEs. In Proceedings of the Advances in Production Management Systems Conference -Global Production Management, Berlin, 6-10 September, 1999.

14.Hudson, M., Bourne, M., Lean, J. and Smart, PA., Only just managing - no time to measure. In Proceedings of Performance Measurement -Past, Present and Future Conference, Cambridge, 19-21 July, 2000.

15.Hudson, M., Smart, A., \& Bourne, M., Theory and practice in SME performance measurement systems. International journal of operations \& production management, 21(8): 1096-1115, 2001.

16.Hussein, M., Gunasekaran, A. and Laitinen, E.K., Management accounting system in Finish service firms. Technovation, 18: 57 67, 1998.

17.Hvolby, H-H., Thorstenson, A., Performance measurement in small and medium-sized enterprises. In Proceeding of the International Conference on Stimulating Manufacturing Excellence in SMEs. Coventry, 17-19 April, 2000.

18.Jennings, P. and Beaver, G., The performance and competitive advantage of small firms: a management perspective. International Small Business Journal, 15: 34-58, 1997.

19.Kaplan, R. and Norton, D., Using the Balanced Scorecard as a strategic management system. Harvard Business Review, Jan-Feb: 75-85, 1996.

20.Lynch, R. and Cross, K., Measure Up! Yardsticks for Continuous Improvement. Cambridge: Blackwell, 1991.

21.Marchini, I., The Small Business Government, vol. 3 - Function management, Genova: ASPI/ INS-EDIT, 1995.

22.Martins, R.A. and Salerno, M.S., Use of new performance measurement system, some empirical findings. In Managing Operations Networks - VI International EurOMA Conference, Venice, Italy, 7-8 June, 1999. 
23. Maskel, B., Performance measures for world class manufacturing. Management Accounting, 5: 32-33, 1989.

24.McAdam, R., Quality models in an SME context. International Journal of Quality and Reliability Management, 17: 305-323, 2000.

25.Neely, A., Mills, L, Richards, H., Gregory, M., Bourne, J. and Kennerley, M., Performance measurement system design: developing and testing a process-based approach. International Journal of Operations and Production Management, 20: 1119-1145, 2000.

26.Neely, A.D. and Mills, J.R, Manufacturing in the UK-Report on a Survey of Performance Measurement and Strategy Issue in UK Manufacturing Companies. London: Manufacturing Engineering Group, 1993.

27.Neubauer, R and Lank, A.G., The Family Business. Its Governance for Sustainability. London: Macmillan, 1998.
28.Noci, G., Accounting and non-accounting based measures of quality-based performances in small firms. International Journal of Operations and Production Management, 15: 78-106, 1995.

29. Storey, D. (1994). Understanding the Small Business Sector. London: Routledge. Suwignjo, P., Bititci, U.S. and Carrie, A.S., Quantitative models for performance measurement systems. International Journal of Production Economics, 64: 231-241, 2000.

30.Tenhunen, J., Rantanen, H. and Ukko, J., SME-oriented Implementation of a Performance Measurement System. Lahti, Finland: Department of Industrial Engineering and Management, Lappeenranta University of Technology, 2001.

31.Welsh, LA., White, J.R, Converging on characteristics of entrepreneurs, In: Vesper, K.H. (ed.), Frontiers of Entrepreneur ship Research. Wellesley, MA: Babson Centre for Entrepreneurial Studies, 1981. 